\title{
E-Beam Generated Plasma Etching for Developing High-Reflectance Mirrors for Far-Ultraviolet Astronomical Instrument Applications
}

\author{
Manuel A. Quijada ${ }^{a}$, David R. Boris ${ }^{b}$, Javier del Hoyo ${ }^{a}$, Edward J. Wollack ${ }^{a}$, Alexander C. \\ Kozen $^{c}$, Scott Walton ${ }^{b}$, and Vivek Dwivedi ${ }^{a}$ \\ ${ }^{a}$ NASA Goddard Space Flight Center, \\ 8800 Greenbelt Rd., Greenbelt, MD 20771, USA; \\ ${ }^{b}$ Plasma Physics Division, U.S. Naval Research Laboratory, \\ 4555 Overlook Ave. SW, Washington, DC 20375, USA \\ ${ }^{c}$ ASEE Postdoctoral Research Associate, U.S. Naval Research Laboratory, U.S. Naval \\ Research Laboratory, \\ 4555 Overlook Ave. SW, Washington, DC 20375, USA
}

\begin{abstract}
Astronomical space telescopes to study astrophysical phenomena from the far-ultraviolet (FUV) to the near infrared (NIR) will require mirror coatings with high reflectance over this entire spectral region. While coatings for the optical and NIR part of the spectrum are fairly well developed with proven performance, the FUV presents significant challenges. The U.S. Naval Research Laboratory (NRL) has developed a processing system based on an electron beam-generated plasma that provides for controlled fluorination and/or etching of surfaces with near monolayer precision and minimal changes to surface morphology. In this paper, we report recent results of samples treated in the NRL Large Area Plasma Processing System (LAPPS) where restoration of the high intrinsic reflectance in the FUV spectral range have been observed of aluminum $(\mathrm{Al})$ mirrors protected with a magnesium di-fluoride $\left(\mathrm{MgF}_{2}\right)$ overcoat. This paper will also extend these studies to other $\mathrm{Al}$ mirrors protected with aluminum tri-fluoride $\left(\mathrm{AlF}_{3}\right)$ in order to realize the high intrinsic reflectance $\mathrm{Al}$ down to $\mathrm{FUV}$ wavelengths $(100-200 \mathrm{~nm})$, while still maintaining the high reflectance in the optical and NIR spectral regions. Laboratory test data and optical diagnostic techniques used to verify surface scattering and durability of selected coatings will be presented. Finally, we will discuss the scalability of the LAPPS etching process in order to realize these high-reflectivity coatings on mirror segments as large as those proposed for the Large Ultraviolet, Optical, and Infrared (LUVOIR) astronomical telescope system (1+meter class).
\end{abstract}

Keywords: FUV reflectance, E-beam plasma, LUVOIR, HabEx, thin film coatings: aluminum, $\mathrm{MgF}_{2}, \mathrm{AlF}_{3}$, $\mathrm{LiF}$

\section{INTRODUCTION}

This paper describes recent advances in state-of-the-art optical coating technology that will be key to providing improvements that are needed to coat the large mirrors planned for advanced NASA mission concepts such as the Large UV/Optical/IR (LUVOIR) Surveyor observatory and the Habitable Exoplanet Imaging Mission (HabEx). ${ }^{1,2}$ Both of these telescope architectures, which will have a spectral coverage from the far-ultraviolet (FUV) to the near-infrared (NIR) spectral ranges $(90-2500 \mathrm{~nm}$ ) and have as goals to unveil fundamental information related to primary NASA mission, such as the history of galaxies (both the Milky Way and its neighbors), the origins of stars and planets, the demographics of planetary systems, and the search for life.

Although aluminum (Al) based reflectors have been identified as a baseline coatings for both LUVOIR and HabEx, the performance of this material is still deficient in the FUV spectral range. ${ }^{3,4}$ Accordingly, improving optical coating technology has been identified as an "Essential Goal" in the technology needs that have been

Send correspondence to M.A.Q.: E-mail: manuel.a.quijada@nasa.gov, Telephone: +1 3012863544 
proposed in the two separate Science and Technology Definition Team (STDT) studies commissioned by NASA to study the feasibility of a LUVOIR or HabEx mission and in preparation for the full reports to be delivered for the 2020 Astrophysics Decadal Survey. Improved reflective coatings for optics in the FUV spectrum could yield dramatically more sensitive instruments and permit more instrument design freedom. ${ }^{5}$ Therefore, obtaining broadband coatings with a performance of at least $50-70 \%$ reflectance below $120 \mathrm{~nm}$ is an immediate, high-priority technology investment area, fundamental to mission feasibility. This paper aims at reporting the development of Al-based broadband optical coatings through a joint effort between the NASA Goddard Space Flight Center (GSFC) and the U.S. Naval Research Laboratory (NRL) where we show improvements in FUV reflectivity of Al-based coatings developed at GSFC that are protected with a single layer of the metal-fluoride $\left(\mathrm{LiF}, \mathrm{MgF}_{2}, \mathrm{AlF}_{3}\right)$ dielectric overcoats ${ }^{6-8}$ in combination with the use of the NRL's proprietary Large Area Plasma processing System (LAPPS), ${ }^{9}$ which is based on an electron beam generated plasma remove oxide and passivate the surface of $\mathrm{Al}$ coatings with a fluoride layer.

In this paper, we report on the efforts to perform fluorination experiments in the LAPPS reactor located at NRL. These experiments were done with a dual purpose. The first was to remove the naturally occurring $\mathrm{Al}_{2} \mathrm{O}_{3}$ layer from oxidized $\mathrm{Al}$ samples followed with a passivation of the surface with a thin $\mathrm{AlF}_{3}$ overcoat.

This approach is motivated by earlier reports ${ }^{10,11}$ that have demonstrated these fluorinations experiments will result in the formation of a thin layer of $\mathrm{AlF}_{3}$, which is a dielectric material with a low refractive index with excellent transparency in the FUV spectral region. The passivation of oxide-free $\mathrm{Al}$ films with $\mathrm{AlF}_{3}$ has the potential to realize the high reflectance of $\mathrm{Al}$ down to cut-off wavelength of the $\mathrm{AlF}_{3}$ compound.

This paper also discuss the feasibility of treating samples consisting of $\mathrm{Al}$ coatings with a metal-fluoride overcoat (such as $\mathrm{LiF}, \mathrm{MgF}_{2}$, or $\mathrm{AlF}_{3}$ ) that have been through a plasma treatments at NRL aimed at either controllably etching the metal-fluoride layer, densifying this layer through a low energy ion bombardment, or homogenizing the fluorine content of the film, all of which should lead to enhanced FUV reflectance performance. The paper is organized as follows: Section 2 provides a background in the state-of-the-art FUV coatings for the next generation UV/Visible space observatory. Section 2.2 gives a description of the LAPPS system at NRL, while Section 2.1 provides a description of the Physical Vapor Deposition (PVD) techniques and advances of FUV coatings developed at GSFC. In the experimental detail section (Section 3), we give a description of the e-beam etching process applied to Al-based coatings, while Sec. 3.2 gives details on instrumentation used for spectral testing including a description of a vacuum ultraviolet (VUV) spectrometer. Section 4 is devoted to present results of these efforts. We start in Sec. 4.1 by presenting results of restoring the pristine reflectance of an aged $\mathrm{Al}+\mathrm{MgF}_{2}$ sample, along with results and discussion on the attempt to thin-out the dielectric layers of a second $\mathrm{Al}$ mirror coatings protected with $\mathrm{MgF}_{2}$. Section 4.2 presents reflectance results on bare-Al samples that were LAPPS treated for the purpose of removing the native oxide layer of bare Al samples and simultaneously perform a passivation of the surface with a thin $\mathrm{AlF}_{3}$ overcoat. The paper ends with some concluding remarks and plans for future work.

\section{ULTRAVIOLET COATINGS FOR THE NEXT GENERATION ULTRAVIOLET/VISIBLE SPACE OBSERVATORY}

A number of experiments have been reported in the literature to improve the deposition of $\mathrm{MgF}_{2}$ and $\mathrm{LiF}$ films that are intended as a protective overcoat for $\mathrm{Al}$ mirrors and interference filters in the FUV) spectral range. ${ }^{4,6}$ To maximize reflection of either the $\mathrm{Al}+\mathrm{MgF}_{2}$ or $\mathrm{Al}+\mathrm{LiF}$ coating pairs, the key is to produce a thin film of $\mathrm{MgF}_{2}$ (or LiF) that is minimally absorbing in the FUV with a thickness of approximately a quarter of the operating wavelength in the 90-180 $\mathrm{nm}$ range. Next section will describe in more details efforts developed at GSFC for enhancing reflectance performance of Al-based coatings in the FUV spectral region.

\subsection{FUV Coating Facility at GSFC}

The GSFC coating facilities have a long heritage of providing coating services for protected Al-based mirrors in support of NASA flagship missions. The most notable examples include several generations of the Hubble Space Telescope (HST) instruments, including the Corrective Optics for Space Telescope Axial Replacement (COSTAR), the Goddard High-Resolution Spectrograph (GHRS), Space Telescope Imaging Spectrograph (STIS), 


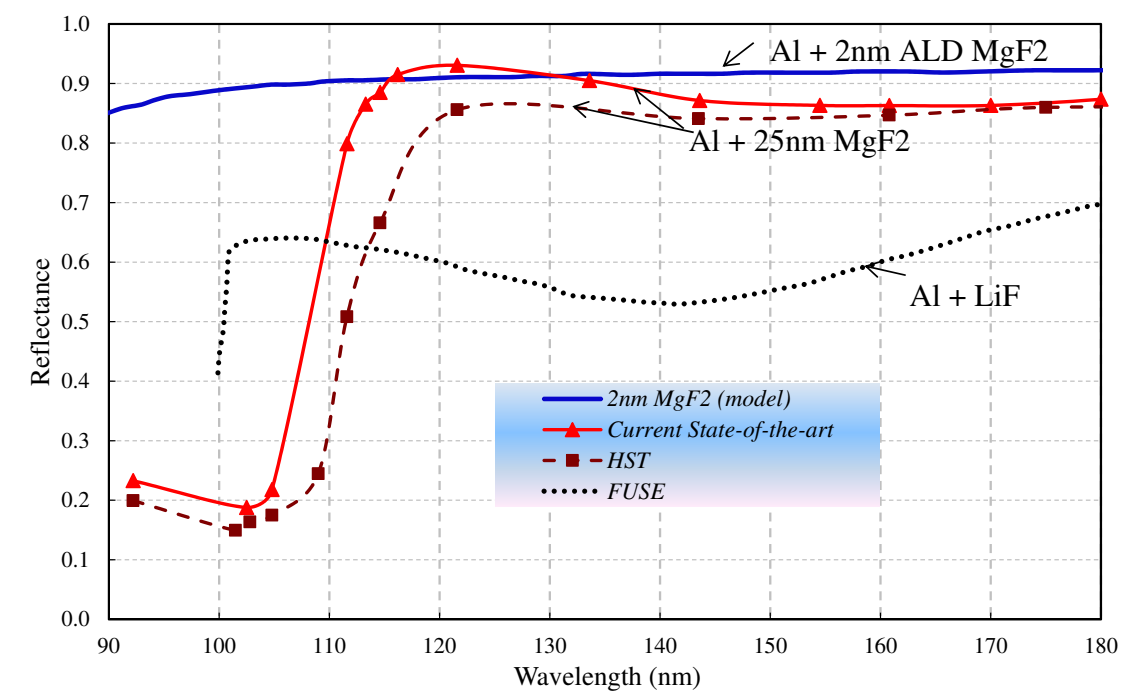

Figure 1. State-of-the-art reflectance performance of $\mathrm{Al}+\mathrm{MgF}_{2}$ mirror coatings in the FUV spectral region.

Cosmic Origin Spectrograph (COS) as well as optics for the FUSE (Far Ultraviolet Spectroscopic Explorer) mission.

There have been recent advances in the production of $\mathrm{Al}$ coatings protected with metal fluoride dielectric films that reach reflectance over $90 \%$ at the Lyman-alpha wavelength $(\lambda=1216 \mathrm{~nm})$. The reason for these gains has been attributed to the deposition of more dense overcoat layers of either $\mathrm{MgF}_{2}$ or LiF with intrinsic cut-off wavelengths closer to the theoretical limit. ${ }^{6}$ The deposition method used to produce these samples is via a physical vapor deposition (PVD) process of both the $\mathrm{Al}$ and the metal fluoride passivation layer, with the key difference being that the latter metal-fluoride layer is done with the substrate at elevated temperature $\left(\approx 260{ }^{\circ} \mathrm{C}\right) \cdot{ }^{7}$

The $\mathrm{Al}$ coating process is based on a Physical Vapor Deposition (PVD) method where the $\mathrm{Al}$ is placed in a resistive bowl (made out of Tungsten) with electrical wires attached to it. A current is passed through this bowl until the $\mathrm{Al}$ melts and evaporates onto substrate to be coated inside the vacuum chamber. One parameter that determines the quality of the deposited $\mathrm{Al}$ is a high vacuum (which is typically maintained from the low $10^{-7}$ Torr to the high $10^{-8}$ Torr range) during the coating process. it is also key to ensure this vacuum chamber would have very low levels of residual water vapor and oxygen in order to minimize the possibility of oxidation of the $\mathrm{Al}$ layer. The $\mathrm{Al}$ deposition rate is maintained at $100 \AA$ per second or higher, in order to obtain a denser $\mathrm{Al}$ film. The next step in the process is to apply a protective layer of a metal-fluoride dielectric such as $\mathrm{MgF}_{2}, \mathrm{LiF}_{\text {, }}$ or $\mathrm{AlF}_{3}$ in order to avoid oxidation of the $\mathrm{Al}$ that would diminish reflectance performance in the FUV range. This is done by first applying an initial $5 \mathrm{~nm}$ layer of the metal-fluoride layer on top of the $\mathrm{Al}$ to protect it from oxidation. A series of halogen quartz heaters inside the chamber are turned on for raising the Al-coated substrate to a temperature of up to $240{ }^{\circ} \mathrm{C}$. The remaining thickness of the metal fluoride layer is done with the substrate at this elevated temperature.

Figure 1 displays reflectance results of $\mathrm{Al}+\mathrm{MgF}_{2}$ coatings that were performed following the steps described above in a vacuum coater that is capable of performing PVD coating processes. The top blue square curve in this figure represents the theoretical prediction of $\mathrm{Al}+\mathrm{MgF}_{2}$, thicknesses of $50 \mathrm{~nm}(\mathrm{Al})$ and $2 \mathrm{~nm}\left(\mathrm{MgF}_{2}\right)$. The curves labeled "HST" and "FUSE" represent the typical reflectance on these two missions prior to launch. The "HST" coating consisted of an Al layer ( $50 \mathrm{~nm}$ ) followed by a dielectric overcoat of $\mathrm{MgF}_{2}(25 \mathrm{~nm})$. The coating prescription for the third triangle (red) symbols curve is the same as that of the "HST" data and it was obtained on a test coupon where the $\mathrm{MgF}_{2}$ deposition was done at elevated $\left(240{ }^{\circ} \mathrm{C}\right)$ as described above. These data provides the highest reflectance (from $32 \%$ to $65 \%$ at $110 \mathrm{~nm}$ ) and it represents the current state-of-the-art in FUV reflectance for $\mathrm{Al}+\mathrm{MgF}_{2}$ coatings done with the "hot" or "3-step" process. ${ }^{6}$ We have also produced $\mathrm{Al}$ coatings protected with $\mathrm{LiF}$ where this metal-fluoride layer was done with the substrate at elevated temperature 


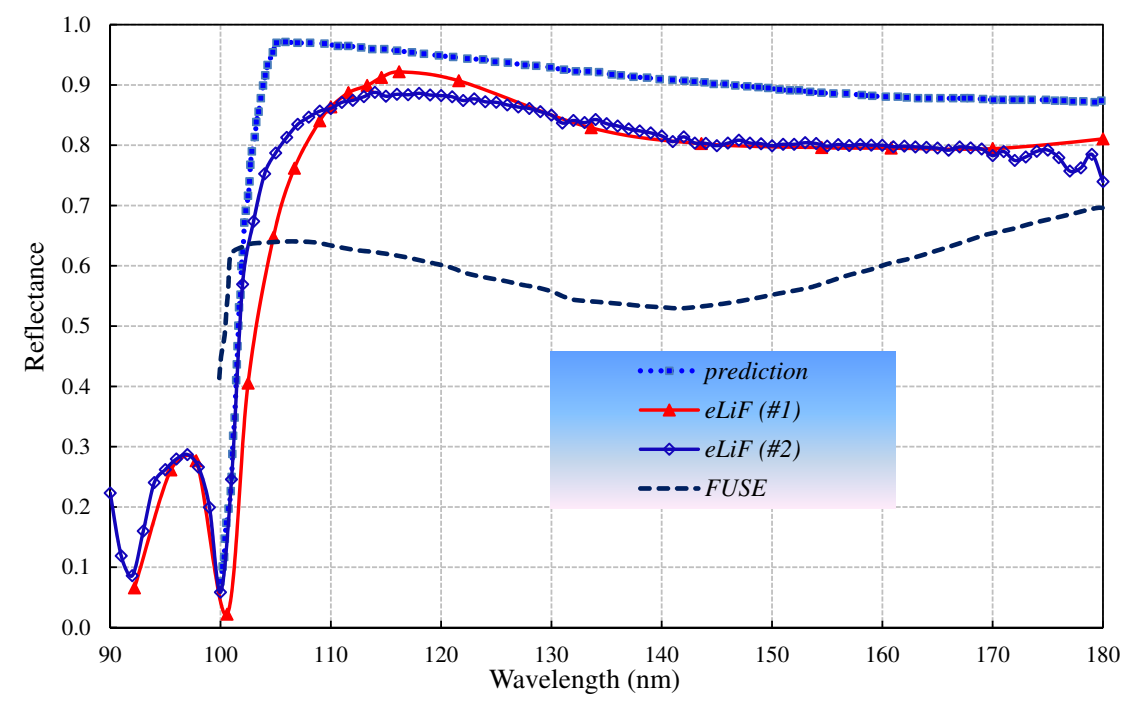

Figure 2. State-of-the-art reflectance performance of $\mathrm{Al}+\mathrm{LiF}$ mirror coatings in the FUV spectral region.

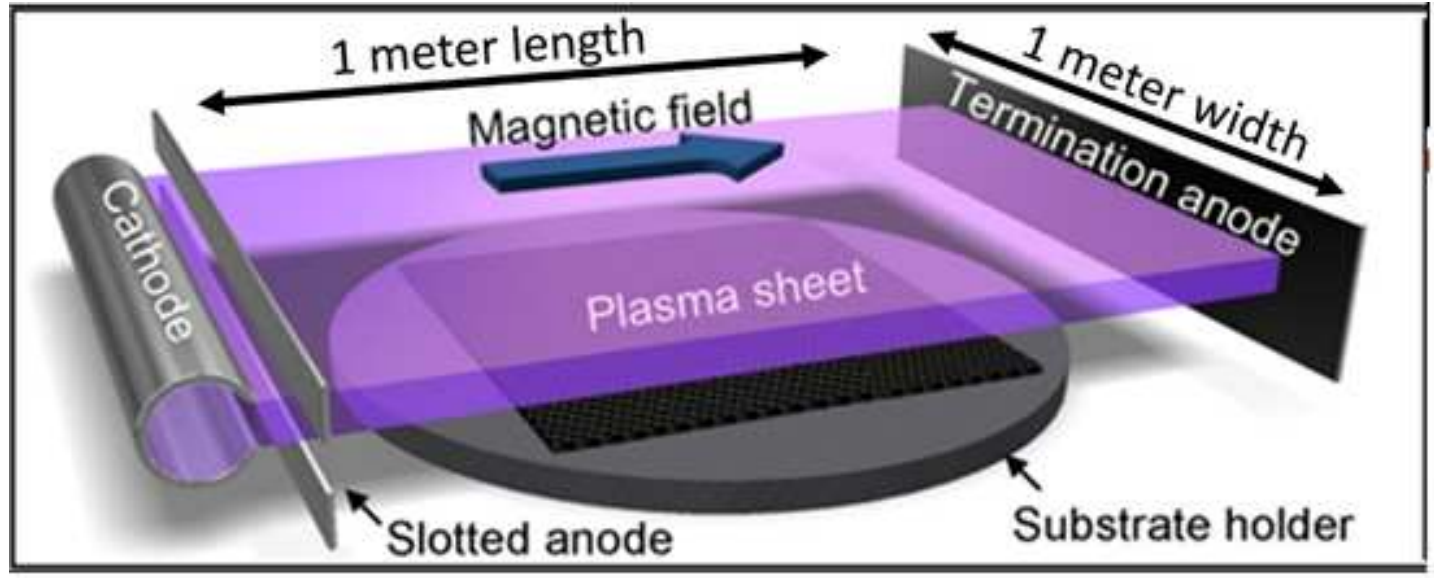

Figure 3. The US Naval Research Laboratory's Large Area Plasma Processing System (LAPPS) facility unique to NRL, based on e-beams, allowing the generation of very large area $\left(>1 \mathrm{~m}^{2}\right)$, highly uniform, low temperature plasmas. The plasma length is determined by beam energy and the plasma width is determined by the length of the hollow cathode. Both length and width can be $>1 \mathrm{~m}$ if desired.

$\left(\simeq 240{ }^{\circ} \mathrm{C}\right)$. These results are shown in Fig. 2 along with a representative curve from the mirror coating used in the FUSE instrument prior to launch. The coating parameters for the eLiF \#1 sample has a total LiF layer thickness of $24 \mathrm{~nm}$, while eLiF \#2 the LiF layer is $21 \mathrm{~nm}$ (the Al layer in both samples is around $45 \mathrm{~nm}$ ). The curve labeled "prediction" was obtained by assuming $50 \mathrm{~nm}$ of Al with a LiF overcoat of $18 \mathrm{~nm}$. The first observation is that both eLiF \#1 and \#2 show a substantial improvement when compared to the "FUSE" results. These reflectance values are the highest ever reported for a $\mathrm{Al}+\mathrm{LiF}$ mirror. ${ }^{7}$ As expected, the thinner the $\mathrm{LiF}$ layer the higher reflectance at lower wavelengths.

\subsection{LAPPS Facility at NRL}

The LAPPS facility was developed nearly two decades ago as a means to generate large area $\left(>1 \mathrm{~m}^{2}\right)$ high-density plasmas for the purposes of materials processing ${ }^{12,13}$ and has received considerable attention recently ${ }^{14}$ as an attractive systems to meet the demands of atomic layer processing. The system makes use of linear hollow cathode electron sources to generate sheet-like electron beams with typical current densities of $1-5 \mathrm{~mA} / \mathrm{cm}^{2}$, and beam 


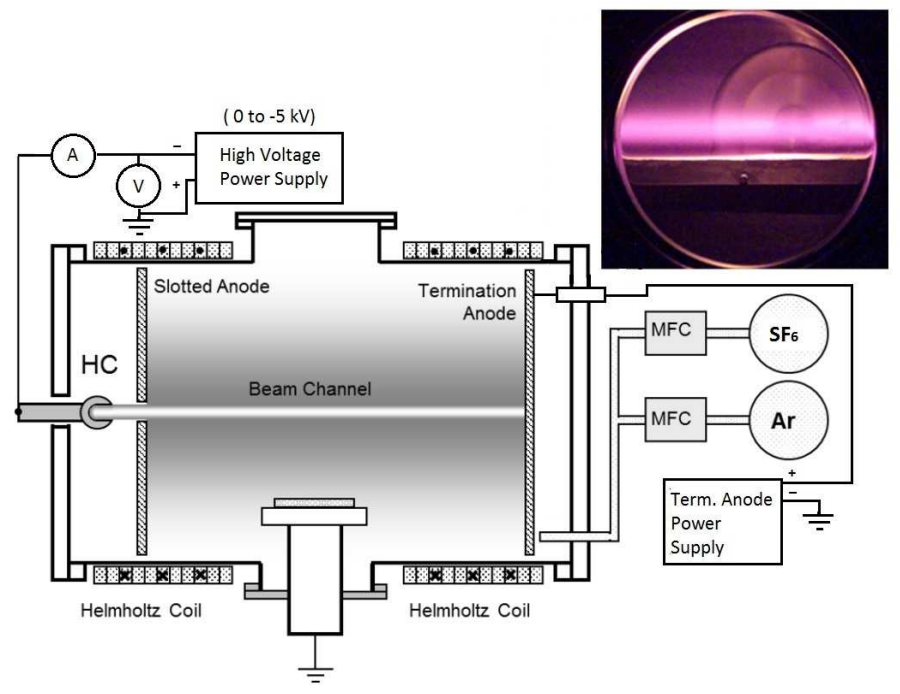

Figure 4. The LAPPS facility employs an electron beam generated plasma. The diagram shows An illustration of the processing reactor used in this work with the radical source shown on the right. The upper right picture shows an image of the plasma through a 6 inch port (after Reference [10]).

energies between $1-5 \mathrm{keV}$. The system is relatively simple, consisting of an e-beam source, entrance aperture through which the beam is injected, termination anode, and magnetic field coils. Materials are introduced on a processing stage that is oriented parallel to the direction of beam propagation and below the beam volume. Co-axial magnetic fields of 100-300 Gauss are used to collimate the e-beam and thus improve uniformity along its length. ${ }^{15}$ The chosen magnetic field strength leaves the plasma ions un-magnetized while aiding in electron confinement. These parameters are sufficient to produce uniform plasma sheets compatible with typical waferscale systems (diameter $>300 \mathrm{~mm}$. LAPPS is able to generate uniform, low electron temperature $\left(\mathrm{T}_{e}\right)$ plasmas with densities in the range of 1010-1012 electrons per $\mathrm{cm}^{3} .{ }^{16}$ The processing stage can either be left at ground (un-biased) resulting in substrate bombardment by very low energy ions $(<5 \mathrm{eV})$ or biased using either DC or $\mathrm{RF}$ voltage to raise the ion energy. Figure 3 illustrates the basic design of an e-beam based plasma processing system.

An important attribute of this system, particularly in the context of thin film processing, is its ability to finely control the ion flux and ion energy distribution (IED) at surfaces adjacent to the plasma. This system is able to deliver ions with energies ranging from $5 \mathrm{eV}$ to as high as $100 \mathrm{eV}$ or greater to both conducting and insulating substrates. This provides a degree of flexibility in ion energy that no other industrial plasma source is able to provide. ${ }^{17}$ In addition, e-beam generated plasmas can be combined with auxiliary methods to generate reactive species to control not only the ion flux but also the ratio of ions to reactive neutrals incident on the surface. ${ }^{18,19}$ Finally, the scaling of electron beam generated plasmas is straightforward, the plasma length is determined by the beam energy (or cathode voltage) and the plasma width is determined by the length of the hollow cathode. Both dimensions can be $>1 \mathrm{~m}$ if desired (see schematic diagram in Fig. 3). The combined abilities to deliver a well-controlled flux species, characterized by a highly tunable ion-to-reactive neutral ratio and very broad incident ion energy range, over a scalable area is a challenging endeavor with conventional, industrial plasma processing tools and well-suited to meet the demands of processing large area, very thin films without damage.

\section{EXPERIMENTAL DETAILS}

This section is devoted to provide experimental details of the approach by first producing $\mathrm{Al}$ coatings with and without a metal-fluoride overcoat and then using the etching and passivation techniques described earlier (namely the treatment using the LAPPS facility at NRL) to realize $\mathrm{Al}$ mirrors with high reflectance performance over the full spectral range including the FUV region. 


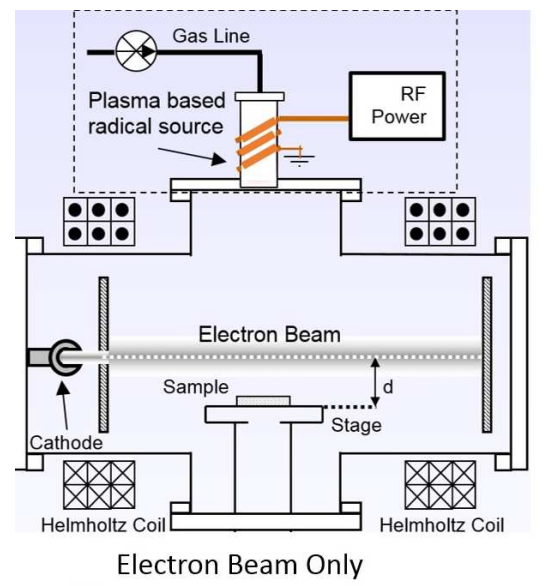

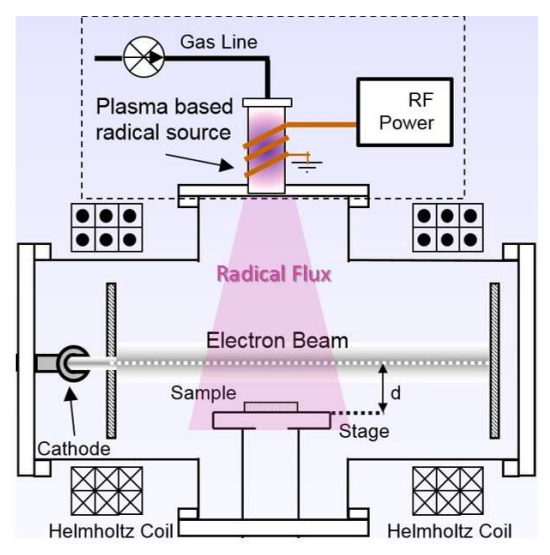

Electron Beam+Radical Source

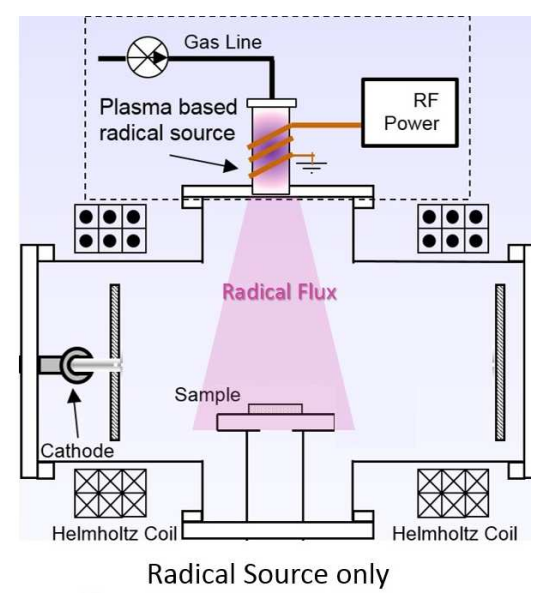

Radical Source only

Figure 5. The three fluorination configurations of e-beam treatment using LAPPS. E-beam treatment only (left); E-beam AND auxiliary F atom source (center); Auxiliary F atom source only (right).

\section{1 e-Beam Plasma Etching/Passivation}

Schematics of the processing systems used in this work are shown in Figs. 4 and 5. For the processing of $\mathrm{Al}+\mathrm{MgF}_{2}$, the plasma processing system (Fig. 4) and its operation for the modification of materials has been previously described in detail elsewhere (see ref. 11 and the references therein) Briefly, the system utilizes a linear hollow cathode immersed in a background of $\mathrm{Ar}$ (purity > 99.9999\%) and $\mathrm{SF}_{6}$ (purity > 99.9999\%). A magnetic field of 150 Gauss was produced by a set of external coils. The samples were placed on a processing stage located $3.0 \mathrm{~cm}$ from the e-beam axis. The stage was grounded and held at room temperature. The plasma potential within the processing volume was controlled by positively biasing the termination anode. This allows for precise control of the kinetic energy of incident ions on the substrate. ${ }^{20}$ Since the surface of the Al thin-film samples is conductive, RF biasing is not required as long as electrical contact between the substrate and processing stage is maintained. The second system, shown in Fig. 5, is described in more detail in Ref. $\left[{ }^{21}\right]$. It employs a cylindrical e-beam, produced by a cylindrical hollow that also allows independent control over the beam current and energy, Otherwise, the plasmas have nominally similar characteristics (except for the geometry). In addition, an ICP plasma source was added to the processing reactor for the purpose of providing a larger $\mathrm{F}$ radical density within the processing environment. When operating, the gases were feed though this source, while all other operating conditions were the same. Figure 5 displays the three fluorination operating configurations that were used for the treatment of performed on protected (oxidized) Al samples.

\subsection{FUV Reflectance Measurements}

The instrument used to measure reflectance as a function of wavelength is a MacPherson Vacuum Ultraviolet (VUV) 225 spectrophotometer. This spectrometer is a one-meter high-vacuum monochromator designed to provide access to the spectral range from $30 \mathrm{~nm}$ to $325 \mathrm{~nm}$ with a 1200 Grooves/mm grating. Effective coverage of the spectral range is dependent upon factors such as the optical coatings, grating efficiency, order-sorting filters, light source and detector. The spectrometer is equipped with a windowless hydrogen-purged light source, which provides discrete $\mathrm{H}_{2}$ emission lines between $90 \mathrm{~nm}$ and $160 \mathrm{~nm}$ and a continuum above these wavelengths. The detector, which is housed inside a sample-holder compartment consists of a photomultiplier cathode tube (PMT) connected to a light-pipe for feeding the light signal coming out of the monochromator. The light pipe has a fluorescence and high quantum efficiency coating of sodium salicylate that is used to convert the FUV radiation into visible light. The maximum emission efficiency of this coating matches that of the PMT sensitivity curve that is close to $420 \mathrm{~nm}$.

The reflectance measurement is performed by first collecting a 100\% reference measurement by moving the detector in front so that it captures all the energy coming from the optical path and source inside the spectrometer. The sample to be measured is then inserted in the optical path of the beam and the detector is positioned to 

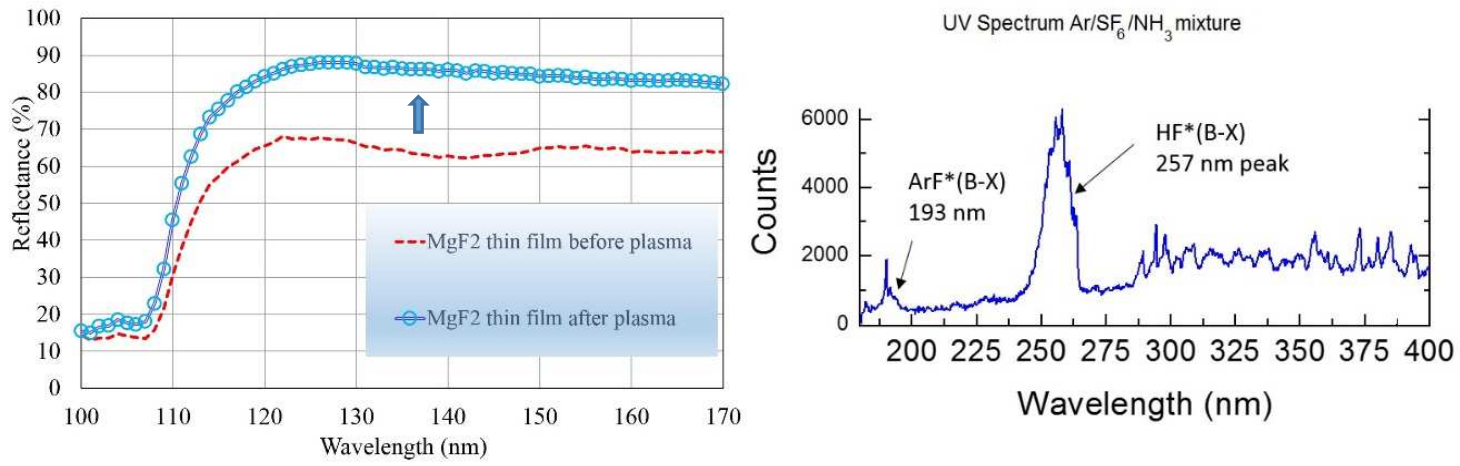

Figure 6. (Left panel) The remarkable regeneration of a 6 year old $\mathrm{MgF}_{2}$ coated $\mathrm{Al}$ mirror to as grown conditions after exposure to the LAPPS plasma employing an $\mathrm{Ar} / \mathrm{SF}_{6} / \mathrm{NH}_{3}$ chemistry. (Right panel) UV spectrum showing HF* emission band at $257 \mathrm{~nm}$ to be the dominant spectral feature of the plasma in the UV range, indicating it is likely a substantial component of the radical flux to the coating surface.
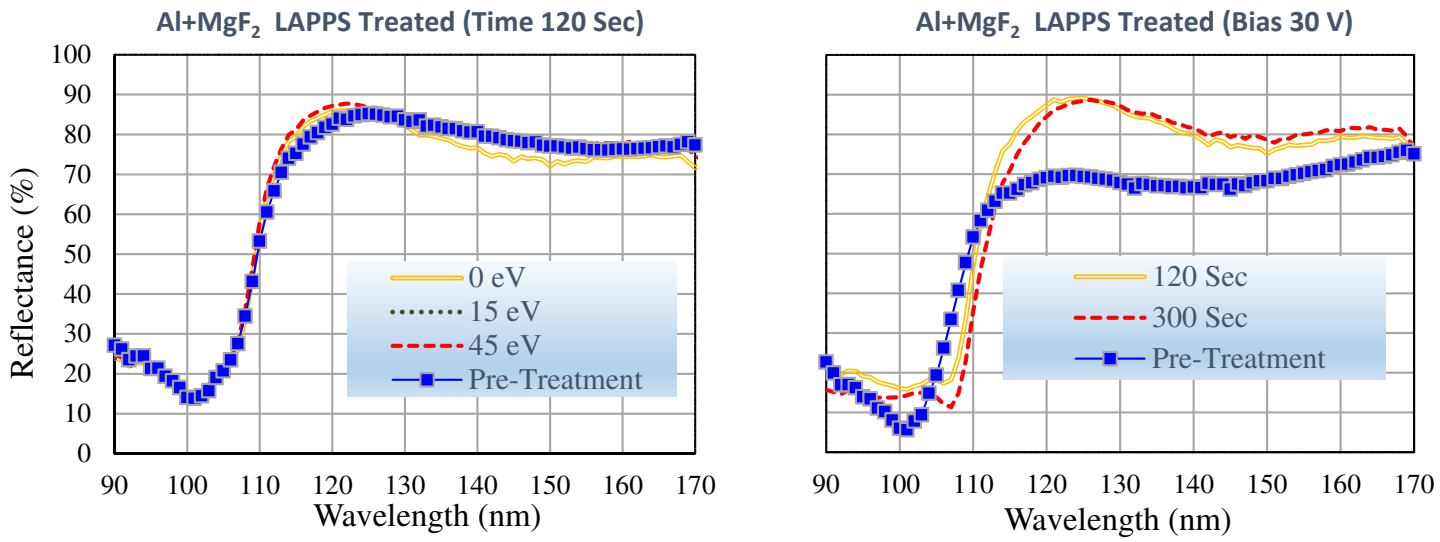

Figure 7. Left panel: The FUV reflectance of two $\mathrm{Al}+\mathrm{MgF}_{2}$ samples that were treated for about 120 seconds does not show a measurable change (with respect to the untreated sample) after LAPPS treatment with 0,15 , and $45 \mathrm{eV}$ ion energies. Right panel: The FUV reflectance of two additional $\mathrm{Al}+\mathrm{MgF}_{2}$ samples that were treated with a constant ion energy bias of $30 \mathrm{~V}$ display changes after being exposed for 120 seconds and 300 seconds respectively.

measured the energy reflected off the surface of the sample (at a near-normal angle of incidence close to $10^{\circ}$ ). The final step is to take the numerical ratio of the signal reflected off the sample to the $100 \%$ reference signal collected above. The resulting ratio provides the absolute reflectance response from the sample without the need of a reference standard.

\section{RESULTS: REFLECTANCE OF LAPPS TREATED SAMPLES}

\section{1 $\mathrm{Al}+\mathrm{MgF}_{2}$}

We will first present results of treating an old $\mathrm{Al}+\mathrm{MgF}_{2}$ sample made in the coating facilities at GSFC in order to demonstrate the capabilities of LAPPS using the system shown in Fig. 5 to process and enhance the qualities of Al mirrors. The approach of treating this sample with LAPPS involved using plasma chemistry that has been shown to generated gas phase HF from benign precursors $\left(\mathrm{Ar} / \mathrm{SF}_{6} / \mathrm{NH}_{3}\right)$ for the purpose of enhancing stoichiometry and thin films properties. This process was not optimized but has demonstrated the ability to enhance the properties $\mathrm{Al}$ mirrors protected with $\mathrm{MgF}_{2}$ as shown in Fig. 6. The reflectance enhancement seen in this figure is quite remarkable, given that this sample was originally made in the year 2011 and due to poor handling its average reflectance had dropped to about $65 \%$ in the $110-170 \mathrm{~nm}$ spectral range. The treatment with LAPPS restored the average reflectance of this sample to its pristine value of $85 \%$ (a $20 \%$ increase) in the 

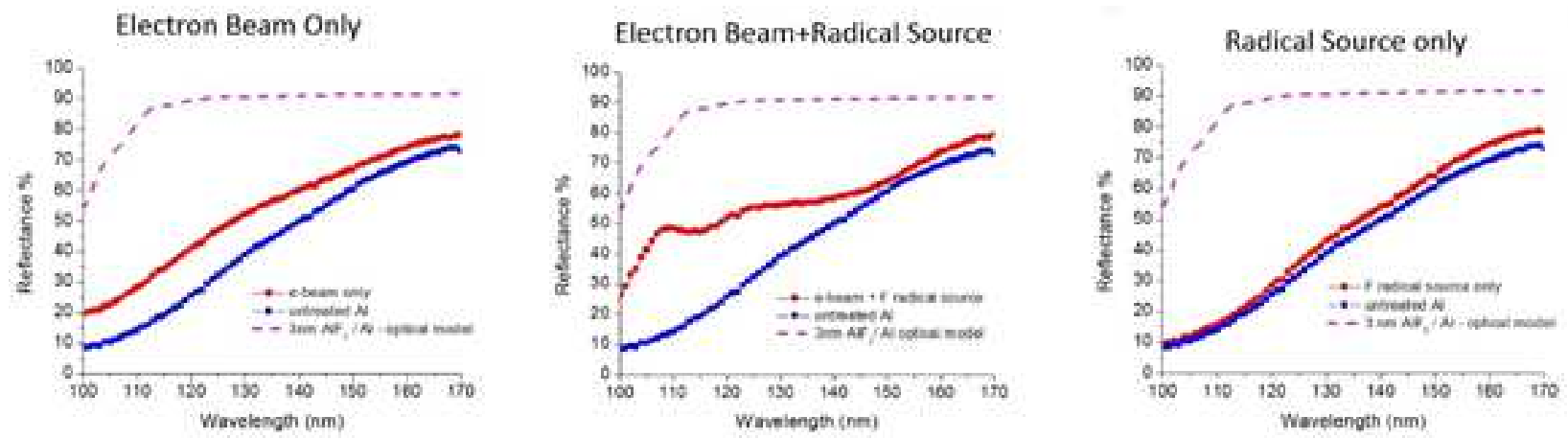

Figure 8. Reflectance before (blue) and after (red) results for native oxide removal and fluorination with e-beam only (left). Reflectance before (blue) and after (red) results for native oxide removal and fluorination with LAPPS AND auxiliary $\mathrm{F}$ atom source (center). Reflectance before (blue) and after (red) results for native oxide removal and fluorination with auxiliary $\mathrm{F}$ atom source only (right). The dash lines in all three plots are theoretical predictions for Al with a thin (3 $\mathrm{nm})$ layer of $\mathrm{AlF}_{3}$.

same spectral range. This process is analogous to a post-cleaning that is able to remove oxide and/or molecular contaminants. We believe that the combined presence of HF and low energy ion bombardment leads to oxygen removal, and possibly smoothing and densification of the $\mathrm{MgF}_{2}$ layer. It may also be possible to produce thinner optical coatings of high quality with LAPPS by using the plasma to controllably etch back thicker optical coatings to the point where their performance is closer to the ideal limit. Figure 7 displays data on a more recently made $\mathrm{AL}+\mathrm{MgF}_{2}$ mirror coatings. The left panel of this figure shows FUV reflectance data that are unchanged (relative to the untreated sample) after two samples were treated for about 120 seconds with two different ion energies of $0 \mathrm{eV}$ and $45 \mathrm{eV}$ respectively (with a simultaneous exposure to an $\mathrm{Ar} / \mathrm{SF}_{6}$ chemistry. On the other hand, the right panel of Fig. 7 shows FUV reflectance data of two additional $\mathrm{Al}+\mathrm{MgF}_{2}$ samples that were LAPPS treated with a constant ion energy of $30 \mathrm{eV}$ for about 120 seconds and 300 seconds respectively. The results in this figure illustrate a a remarkable increase in the reflectance over the whole range and specially reaching a value close to $90 \%$ at the Lyman alpha wavelength of $121.6 \mathrm{~nm}$.

\subsection{Oxide Removal and Passivation of Bare Al}

Figure 8 shows the results of treating three bare $\mathrm{Al}$ samples with different plasma treatments. These results show that in addition to enhancing the properties of FUV optical coatings, LAPPS has also shown promise as a tool to passivate bare, oxidized $\mathrm{Al}$ mirrors by etching away the native oxide layer on as-grown $\mathrm{Al}$ thin films and replacing that oxide layer with an $\mathrm{AlF}_{3}$ passivation layer in a single-step process.

We achieved this by combining an auxiliary plasma-based atomic F source with the LAPPS plasma. This configuration allowed the controlled removal of native oxide layer of an unprotected $\mathrm{Al}$ film while simultaneously passivating the surface with a layer of $\mathrm{AlF}_{3}$. Importantly, this feat could only be achieved with the combination of LAPPS and the auxiliary source of $\mathrm{F}$ atoms as shown in the center panel of Fig. 8. This combination offers a level of control over both radicals and ions that is difficult to achieve in other plasma processing system offers. Atomic fluorine flux at the substrate is controlled by modulating the power or gas flow on the up-stream, inductively coupled plasma used to dissociate the process gas before entering the LAPPS system. Control of ion flux at the substrate is achieved through varying the electron beam current emanating from the hollow cathode. In Fig. 8 (center panel), we demonstrate an enhancement in reflectivity at $110 \mathrm{~nm}$ from $<20 \%$ for the as grown $\mathrm{Al}$ coating to $48 \%$ after plasma treatment. The thickness of the $\mathrm{AlF}_{3}$ layer is comparable to the original oxide layer (1-5 nm). Ex situ evaluation of the sample in the center plot in this figure with X-Ray Photo-electron Spectroscopy (XPS) indicated that the top $5 \mathrm{~nm}$ of the $\mathrm{Al}$ mirror has the stoichiometry of $\mathrm{AlF}_{3}$ with a residual oxygen impurity of roughly $9 \%$. We attribute the oxygen impurity to a quartz $\left(\mathrm{SiO}_{2}\right)$ window incorporated into the auxiliary plasma based radical source and this likely prevented reaching reflectance values closer to the theoretical prediction shown in the same figure for an $\mathrm{Al}$ film with $3 \mathrm{~nm}$ of $\mathrm{AlF}_{3} \cdot{ }^{11}$ 


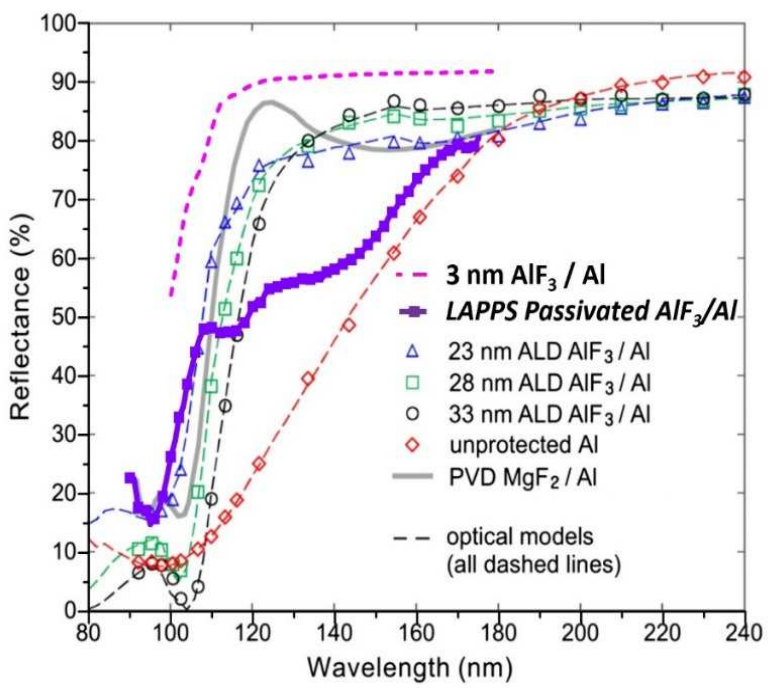

Figure 9. The FUV reflectance curves for a number of state-of-the art ALD coatings [Ref. 14] of AlF3 are shown alongside the FUV reflectance of LAPPS passivated sample (After Ref.[22]).

To put these results in context, in Fig. 9, we compare them with a number of reflectivity characterizations for thin film coatings of $\mathrm{AlF}_{3}$ on $\mathrm{Al}$ deposited using atomic layer deposition (ALD). ${ }^{22}$ These results show that while the LAPPS treated samples shown in Fig. 8 did not reach the predicted FUV reflectivity of $80-90 \%$ range near $120 \mathrm{~nm}$ (due to possibly oxygen contamination) the LAPPS sample actually exceeds the reflectance of the state-of-the-art ALD films at wavelengths below $105 \mathrm{~nm}$.

The choice of the process conditions used in the experiments reported here were driven by the competing needs to both remove the native oxide layer from the surface of the $\mathrm{Al}$ thin film while also leaving the reflective qualities of the thin film either unchanged or enhanced. Thus low ion bombardment energies were chosen for the $\mathrm{Ar}$ clean step to ensure minimal roughening of the $\mathrm{Al}$ thin film, with the hope that the $30 \mathrm{eV}$ ions would be sufficient to remove the $2-4 \mathrm{~nm}$ thick native oxide layer from the bare $\mathrm{Al}$ sample. The subsequent exposure to the $\mathrm{Ar} / \mathrm{SF}_{6}$ mixture was intended to provide a very low energy flux of $\mathrm{F}_{2}^{+}$, and $\mathrm{SF}_{X}^{+}$ions combined with exposure to $\mathrm{F}$, and $\mathrm{F}_{2}$ radicals to generate an $\mathrm{AlF}_{3}$ surface passivation layer. ${ }^{23}$

\section{CONCLUSIONS}

In conclusion, this paper studied the feasibility of using the LAPPS process developed at NRL of using a lowtemperature electron plasma beam to etch away the native oxide layer from bare $\mathrm{Al}$ coatings (prepared in the coating facilities at GSFC) in order to passivate the surface with a thin $\mathrm{AlF}_{3}$ layer. The LAPPS process was also used in a demonstration to restore the pristine reflectance of a six years old $\mathrm{Al}+\mathrm{MgF}_{2}$ sample. We also explored the feasibility of etching and densifying the top layer dielectric overcoat of $\mathrm{Al}$ test samples protected with an $\mathrm{AlF}_{3}$.

The LAPPS etching experiment on bare Al mirrors yielded an increase in FUV reflectivity from 10\% (preexposure) to around $48 \%$ (post-exposure) at $110 \mathrm{~nm}$. Ex-situ analysis using XPS techniques on the surface of these samples indicated that the top $5 \mathrm{~nm}$ has the stoichiometry of $\mathrm{AlF}_{3}$ with a residual oxygen impurity of roughly 9\%. This oxygen impurity is attributed to unintended etching of a quartz $\left(\mathrm{SiO}_{2}\right)$ window that is part of the auxiliary plasma based radical source. This oxygen impurity may have likely prevented reaching reflectance values closer to the theoretical prediction of an $\mathrm{Al}$ coating with about $3 \mathrm{~nm}$ of pure $\mathrm{AlF}_{3}$. As part of future work, we plan to upgrade the auxiliary radical source to one using a sapphire window rather than a quartz window. This would eliminate any oxygen impurities resulting from etching of the quartz tube (a common problem in $\mathrm{F}$ based chemistries) and would likely result in a much higher purity $\mathrm{AlF}_{3}$ layer on the surface of the $\mathrm{Al}$ coating. 
The etching experiments of a protected $\mathrm{Al}$ sample $\left(\mathrm{Al}+\mathrm{MgF}_{2}\right)$ that had its reflectance degraded to about $65 \%$ over a six year period was restored to the average reflectance value of $84 \%$ in the $115-170 \mathrm{~nm}$ range and this is similar to when sample was freshly made. These results and the scalability of the LAPPS process would open the possibility of using the LAPPS facility as a tool to clean and etch away contaminants that may affect the reflectance performance of mirrors in the FUV spectral range.

\section{ACKNOWLEDGMENTS}

The authors wish to thank Felix Threat and Emrold Gray (NASA-GSFC/551) for their support in the coating activities of the enhanced $\mathrm{Al}+\mathrm{LiF}(\mathrm{eLiF})$ samples. This work has been performed through a joint collaboration between the Optics Branch (Code 551) at the Goddard Space Flight Center (GSFC) and the Plasma Physics Division at the Naval Research Laboratory. Financial support is through a NASA Astrophysical Research and Analysis (APRA) grant No. 15-APRA15-0103 and a GSFC Internal Research and Development Program (IRAD) grant. DRB, SGW, and ACK were also supported via the Naval Research Laboratory Base Program and the Office of Naval Research. ACK appreciates the support of the American Society for Engineering Education (ASEE) postdoctoral research associate program.

\section{REFERENCES}

[1] M.R. Bolcar, K. Balasubramanian, J. Crooke, L. Feinberg, M. Quijada, B.J. Rauscher, D. Redding, N. Rioux, S. Shaklan, H. Philip Stahl, C.M. Stahle, H. Thronsona, "A Technology Development Plan for a Future Large-Aperture Ultraviolet-Optical-Infrared Space Telescope," Proc. SPIE 9602 (2016).

[2] B. Mennesson, S. Gaudi, S. Seager, K. Cahoy, S. Domagal-Goldman, L. Feinberg, O. Guyon, J. Kasdin, C. Marois, D. Mawet, T. Motohide, D. Mouillet, T. Prusti, A. Quirrenbach, T. Robinson, L. Rogers, P. Scowen, R. Somerville, K. Stapelfeldt, D. Sterna, M. Still, M. Turnbull, J. Booth, A. Kiessling, G. Kuan, K. Warfield," "The habitable exoplanet (habex) imaging mission: preliminary science drivers and technical requirements," in [Space Telescopes and Instrumentation 2016], MacEwen, H. A., ed., Space Telescopes and Instrumentation 2016: Optical, Infrared, and Millimeter Wave 9904 (2016).

[3] R.A.M. Keski-Kuha, J.F. Osantowski, G.M. Blumenstock, J.S. Gum, C.M. Fleetwood, D.B. Leviton, T.T. Saha, J.G. Hagopian, J.L. Tveekrem, and G.A. Wright, "High reflectance coatings and materials for the extreme ultraviolet," Proc. SPIE 2428, 294-306 (1995).

[4] W.R. Hunter, J.F. Osantowski, and G. Hass, "Reflectance of aluminum overcoated with $\mathrm{MgF}_{2}$ and $\mathrm{LiF}$ in the wavelength region from $1600 \AA$ to $300 \AA$ at various angles of incidence," Appl. Opt. 10, 540 (1971).

[5] J. Tumlinson et al., "Unique Astrophysics in the Lyman Ultraviolet," ArXiv e-prints (2012).

[6] M.A. Quijada, S. Rice, and E. Mentzell, "Enhanced $\mathrm{MgF}_{2}$ and LiF Over-coated Al Mirrors for FUV Space Astronomy," Proc. SPIE $\mathbf{8 4 5 0}$ (2012).

[7] M. A. Quijada, J. Del Hoyo, and S. Rice, "Enhanced far-ultraviolet reflectance of $\mathrm{MgF}_{2}$ and LiF over-coated Al mirrors," 9144, 91444G (2014).

[8] B. Fleming, M. Quijada, J. Hennessy, A. Egan, J. Del Hoyo, B.A. Hicks, J. Wiley, N. Kruczek, N. Erickson, K. France, "Advanced environmentally resistant lithium fluoride mirror coatings for the next generation of broadband space observatories," Applied Optics 56(36), 9941-9950 (2017).

[9] R. A. Meger, R. F. Fernsler, M. Lampe, W. Manheimer, "large area plasma processing system," U.S. Patent $5,874,807(1999)$.

[10] K. Roodenko, O. Seitz, Y. Gogte, J.-F. Veyan, X.-M. Yan, and Y. J. Chabal, "Modification of the Adhesive Properties of $\mathrm{XeF}_{2}$-Etched Aluminum Surfaces by Deposition of Organic Self-Assembled Monolayers," $J$. Phys. Chem. C 114, 2256622572 (2010).

[11] K. Roodenko, M. D. Halls, Y. Gogte, O. Seitz, J.-F. Veyan, and Y. J. Chabal, "Nature of Hydrophilic Aluminum Fluoride and Oxyaluminum Fluoride Surfaces Resulting from XeF2 Treatment of $\mathrm{Al}$ and $\mathrm{Al}_{3}$," J. Phys. Chem. C 115, 2135121357 (2011).

[12] W. M. Manheimer, R. F. Fernsler, M. Lampe, R. A. Meger, "Theoretical overview of the large-area plasma processing system (LAPPS)," Plasma Sources Sci. Technol. 9, 370 (2000).

[13] R. A. Meger, R. F. Fernsler, M. Lampe, W. Manheimer, "Theoretical overview of the large-area plasma processing system (LAPPS)," Plasma Sources Sci. Technol. 9, 370 (2000). 
[14] I. Adamovich et al., "The 2017 Plasma Roadmap: Low temperature plasma science and technology," $J$. Phys. D: Appl. Phys 50, 323001 (2017).

[15] G. M. Petrov, D. R. Boris, Tz. B. Petrova, and S. G. Walton Journal of Vacuum Science 83 Technology A 34, $021302(2016)$.

[16] G.M. Petrov, D.R. Boris, E.H. Lock, T.B. Petrova, R.F. Fernsler, S.G. Walton, "The influence of magnetic field on electron beam generated plasmas," J. Phys. D. Appl. Phys. 48, 275202-8 (2015).

[17] S. G. Walton, D. R. Boris, S. C. Hernandez, E. H. Lock, Tz. B. Petrova, G. M. Petrov, and R. F. Fernsler, "Electron beam generated plasmas for ultra low te processing,", ECS J. Solid State Sci. Technology 4(6), N5033-N5040 (2015).

[18] L. Dorf et. al., "Etching with atomic precision by using low electron temperature plasma," J. Phys. D: Appl. Phys. 50, 274003 (2017).

[19] D. R. Boris, J. Del Hoyo, M. A. Quijada, and S. G. Walton, "In preparation,"

[20] S.G. Walton, C. Muratore, D. Leonhardt, R.F. Fernsler, D.D. Blackwell, and R.A. Meger, "Electron beamgenerated plasmas for materials processing," Plasma Source Sci. Technol. 6 (1-2), 40 (2004).

[21] S. Rauf, A. Balakrishna, A. Agarwal, L. Dorf, K. Collins, D.R. Boris, and S.G. Walton, "3-Dimensional Model of Electron Beam Generated Plasma," Plasma Source Sci. Technol. 26, 065006-12 (2017).

[22] J. Hennessy, K. Balasubramanian, C. S. Moore, A. D. Jewell, S. Nikzad, K. France, and M. Quijada, "Advanced environmentally resistant lithium fluoride mirror coatings for the next generation of broadband space observatories," Journal of Astronomical Telescopes, Instruments, and Systems 2(4), 041206 (OctDec 2016).

[23] D. R. Boris, Tz. B. Petrova, G. M. Petrov, and S. G. Walton, "Atomic fluorine densities in electron beam generated plasmas: A high ion to radical ratio source for etching with atomic level precision," Journal of Vacuum Science $\&$ Technology A 35, 01A104 (2017). 